\title{
COMPARISON OF IMMUNOEXPRESSION OF VASCULAR ENDOTHELIAL GROWTH FACTOR IN ANGIOGENESIS USING DIFFERENT ANTIGEN RETRIEVAL AND DETECTION METHODS
}

\author{
Aktas $\mathrm{RG}^{*}$, Ozacmak VH**, Guven $\mathrm{A}^{* * *}$ \\ *Histology and Embryology Faculty of Medicine, Zonguldak Karaelmas University. \\ Zonguldak, Turkey; \\ ${ }^{* *}$ Physiology Department, Faculty of Medicine, Zonguldak Karaelmas University. \\ Zonguldak, Turkey; \\ ${ }^{* * *}$ Histology and Embryology Department, Faculty of Medicine, Izzet Baysal University, \\ Duzce, Turkey
}

The introduction of antigen retrieval techniques has dramatically improved the sensitivity of immunohistochemical detection of various antigens in formalin-fixed, paraffinembedded tissues. Using different buffers, heating sections and pressure-cooking procedures are the most effective antigen retrieval methods reported to date. Although extensive efforts have been made to optimize the immunostaining procedures using these methods, previous studies have not led to a standard protocol applicable to all antibodies .

The purpose of the present study is to compare immunoexpression of vascular endothelial growth factor in 14 cases of new vessel formation areas, and to verify the effect of different antigen retrieval solutions and detection systems.

Immunoperoxidase was performed on paraffin sections using anti-VEGF as a primary antibody. Sections from each cases were stained by using following protocols: (1) Sections from paraffin-embedded tumor samples were immunostained, using standard immunohistochemical protocols without antigen retrieval. (2)Heat-induced antigen retrieval was performed using citrate buffer $(\mathrm{pH}$ 6.0) or (3) Heat-induced antigen retrieval was performed using PBS buffer ( $\mathrm{pH} 7.3)$. (4) Citrate buffer ( $\mathrm{pH}$ 6.0)was applied without heating. (5)The sections were left in the primary antibody overnight. (6)Pressure cooking procedure was applied on sections as described previously. (7)Primary antibody was omitted as negative control. (8) Sections from ductal breast carcinoma were stained by using the same protocols above as positive controls. Detection was achieved using UltraVision detection system and labeled streptavidin-biotin peroxidase complex. Reaction was semiquantified from 0 to 4 .

There were no significant difference between the first, third, fourth, fifth and sixth group. Immunostaining density were highly increased after using heated citrate buffer(second group). However; case weaker reactivity was observed in this group. There were no staining on the sections from the negative control group; while staining density did not show any significant difference on the sections from the positive control group after the application of different treatments. 
Our results showed that immunostaining the sections with antibody specific for VEGF gave the best results after the treatment with heated citrate buffer ( $\mathrm{pH}: 6.0)$. Heat treatment alone or pressure cooking procedure did not help to increase the labeling for VEGF. Several cases showed weak staining in spite of antigen retrieval treatment. Staining density on the sections from ductal breast carcinoma were similar on all sections after the application of different protocols described above and did not need any retrieval treatment.

We conclude that VEGF expression on tissues increases after using heated citrate buffer as a antigen retrieval method. A major adverse effect of formalin as a fixative is the concealing of tissue antigens by protein cross-linking. We observed that on the sections of several cases, which has poor staining after all different antigen retrieval treatments. The sucsess of the treatment also depends on the type of the tissues and the antibodies. Laboratories might try different retrieval methods if the acquired results were not observed after using standart immunostaining protocols. Citrate ( $\mathrm{pH} \mathrm{6.0)} \mathrm{buffer} \mathrm{used} \mathrm{in}$ conjunction with the heat pre-treatment is strongly recommended for standard routine use.

\section{References}

[1] S Namimatsu et al. J Histochem Cytochem. 2005 Jan;53(1):3-11.

[2] S Kim et al. J Mol Histol. 2004 May;35(4):409-16.

[3] A Ahmed et al. Pediatr Dev Pathol. 2004 Jul-Aug;7(4):342-7. Epub 2004 Jun 17.

[4] J Vassallo et al. Appl Immunohistochem Mol Morphol. 2004 Jun;12(2):177-82.

[5] T Boenisch. Appl Immunohistochem Mol Morphol. 2004 Jun;12(2):172-6.

[6] H Ino. J Histochem Cytochem. 2004 Sep;52(9):1209-17.

[7] Z Kahveci et al. Biotech Histochem. 2003 Apr;78(2):119-28. 